\title{
Solving multi-objective scheduling problems-An integrated systems approach
}

\author{
Martin Josef Geiger \\ Lehrstuhl für Industriebetriebslehre, Universität Hohenheim, D-70593 Stuttgart, \\ Germany, mjgeiger@uni-hohenheim.de
}

\begin{abstract}
In the past, numerous approaches have been formulated either for approximating Pareto-optimal alternatives or supporting the decision making process with an interactive multi criteria decision aiding methodology. The article on the other hand presents an integrated system for the resolution of problems under multiple objectives, combining both aspects. A method base of metaheuristics is made available for the identification of optimal alternatives of machine scheduling problems, and the selection of a most preferred solution is supported in an interactive decision making procedure.

As the system is aimed at end users, a graphical interface allows the easy adaptation of metaheuristic techniques. Contrary to existing software class libraries, the system therefore enables users with little or no knowledge in the mentioned areas to successfully solve scheduling problems and customize and test metaheuristics.

After successfully competing in the finals in Ronneby (Sweden), the software has been awarded the European Academic Software Award 2002 (http://www. easa-award.net/, http://ww.bth.se/llab/easa_ 2002.nsf).
\end{abstract}

Key words: Multi-Objective Optimization, Multi-Objective Metaheuristics, Decision Support System, Scheduling

\section{Introduction}

In general, the resolution of multi-objective problems is twofold. First, optimal solutions need to be identified by means of some algorithmic approach. For a given problem $I$ having a set of feasible alternatives $\mathcal{X}$ and a set of optimality criteria $G(x)=\left(g_{1}(x), \ldots, g_{k}(x)\right), x \in \mathcal{X}$, optimality of alternatives is in the light of conflicting optimality criteria here understood in the sense of Pareto-optimality [20] as further described in Definition 1 and 2. Without loss of generality, a minimization of the objective function values is assumed here.

Definition 1 (Dominance). An objective vector $G(x), x \in \mathcal{X}$ is said to dominate a vector $G\left(x^{\prime}\right), x^{\prime} \in \mathcal{X}$ if and only if $g_{i}(x) \leq g_{i}\left(x^{\prime}\right) \forall i=1, \ldots, k \wedge \exists i$ | $g_{i}(x)<g_{i}\left(x^{\prime}\right)$. The dominance of $G(x)$ over $G\left(x^{\prime}\right)$ is denoted with $G(x) \prec$ $G\left(x^{\prime}\right)$.

Please use the following format when citing this chapter:

Geiger, M.J., 2006, in IFIP International Federation for Information Processing, Volume 217, Artificial Intelligence in Theory and Practice, ed. M. Bramer, (Boston: Springer), pp. 493-502. 
Definition 2 (Efficiency, Pareto-optimality, Pareto set). An objective vector $G(x), x \in \mathcal{X}$ is said to be efficient, if and only if $\neg \exists x^{\prime} \in \mathcal{X} \mid G\left(x^{\prime}\right) \prec$ $G(x)$. The corresponding alternative $x$ is called Pareto-optimal, the set of all Pareto-optimal alternatives the Pareto set $P$.

The second step of the resolution of multi-objective problems is the selection of a most preferred alternative $x^{*} \in P$, involving a single human decision maker or even a group of people.

Search and decision making can be combined in three general ways [6].

1. A priori: The decision maker states his/her preferences allowing the construction of a utility function and the successive resolution of the resulting mono-criterion problem of maximizing the overall utility.

2. Interactive: The resolution of the problem alternates between search and decision making, successively revealing the preferences of the decision maker.

3. A posteriori: The choice of a most preferred alternative is performed after the determination of all optimal solutions.

Over the years, numerous concepts have been proposed to support both aspects of search and decision making. Most interactive methods are based on goal programming [13] or reference point approaches [23] and allow the successive refinement of the decision makers' preferences. An overview is e.g. given by VINCKE [21].

Besides methodological progress, implementations of algorithms have been made freely available on the world wide web. For genetic algorithms for example, an archive is maintained under http://www . aic.nrl.navy.mil/galist/src/. The particular case of multi-objective optimization has been addressed by several researchers, and an overview about implemented source code is maintained by COELlo Coello on the EMOO webpage, http://www.lania.mx/ ccoello/EMOO/EMOOsoftware.html. Unfortunately however, most implementations require a throughout understanding of the underlying methodologies and techniques in order to be reused and adapted to particular problem domains. This can impose a problem in teaching and demonstration work, when non-experts are required to interact with the computer programs. Here, implementations are required similar to established computer user interfaces with which the users are familiar. Only very recently, components are being developed that allow the visualization of the outcomes of multi-objective optimization problems, one example being GUIMOO by CAHON, VAN DEN HEKKE and SEYNHAEvE. Integrated systems however, combining both search and decision making are to our knowledge not freely available yet.

The paper is organized as follows. Section 2 presents an integrated system for multi-objective optimization and decision making, using the example of scheduling under multiple objectives. The problem is well-known from operations research and computer science and is of high practical value with applications in many areas [15]. Results obtained with the system are presented in Section 3 , and conclusions and discussion are given in Section 4 . 


\section{A metaheuristic system for multi-objective scheduling}

\subsection{The addressed problem}

Machine scheduling considers in general the assignment of a set of resources (machines) $\mathcal{M}=\left\{M_{1}, \ldots, M_{m}\right\}$ to a set of jobs $\mathcal{J}=\left\{J_{1}, \ldots, J_{n}\right\}$, each of which consists of a set of operations $J_{j}=\left\{O_{j 1}, \ldots, O_{j o_{j}}\right\}$ [4]. The operations $O_{j k}$ typically may be processed on a single machine $M_{i} \in \mathcal{M}$ involving a nonnegative processing time $t_{j k}$. Usually, precedence constraints are defined among the operations of a job, reflecting its technical nature of processing. Other important aspects that frequently have to be taken into consideration are release dates and due dates of jobs.

A solution $x \in \mathcal{X}$ to the problem, a so called schedule, assigns start and end times for the operations with respect to the defined constraints of the problem.

While first approaches to machine scheduling consider optimality of schedules for a single objective function, multi-objective formulations of the problem have become increasingly of importance in the last years [18]. As these criteria are often conflicting, not a single but a whole set of solutions may be regarded as optimal in the sense of Pareto-optimality, introduced earlier in Definition 2, and the resolution of the problem lies in the identification of all $x \in P$.

Various optimality criteria are based on the completion times $C_{j}$ of the jobs $J_{j}$ in the schedule. The most prominent to mention is the minimization of the maximum completion time (makespan) $C_{\max }=\max \left\{C_{1}, \ldots, C_{n}\right\}$. Another objective is the minimization of the sum of the completion times $C_{s u m}=\sum_{j=1}^{n} C_{j}$. Both measures implicitly try to optimize cost of production by minimizing the production time of the jobs.

In many situations, due dates $d_{j}$ are present for each job $J_{j}$ which define a preferable or required time of job completion. It is here possible to compute due date violations in the form of tardiness values $T_{j}=\max \left\{C_{j}-d_{j}, 0\right\}$. Usual optimality criteria based on this consideration are the minimization of the maximum tardiness $T_{\max }=\max \left\{T_{1}, \ldots, T_{j}\right\}$, the minimization of the total tardiness $T_{\text {sum }}=\sum_{j=1}^{n} T_{j}$, and the minimization of the number of tardy jobs $U=\sum_{j=1}^{n} U_{j}$ where $U_{j}=1$ if $T_{j}>0,0$ otherwise.

In terms of machine efficiency, idle times $I_{i}$ of the machines $M_{i}$ may be considered up to the completion of the last job on $M_{i}$. Possible optimality criteria are therefore the minimization of the maximum machine idleness $I_{\max }=$ $\max \left\{I_{1}, \ldots, I_{m}\right\}$, and the minimization of the total machine idleness $I_{\text {sum }}=$ $\sum_{i=1}^{m} I_{i}$.

An important factor for the resolution of the scheduling problem is the regularity of the functions [5]. It is here possible to represent an optimal schedule as a permutation of operations, corresponding to the position of the job in the sequence of production. An interpretation of the permutations is possible by computing a schedule with respect to the given job sequence, assuming earliest possible execution of the operations [7]. 


\subsection{Components}

For the resolution of multi-objective production scheduling problems, the integrated system MOOPPS has been implemented. As illustrated in Figure 1, the system consists of different components for the resolution of the problem.

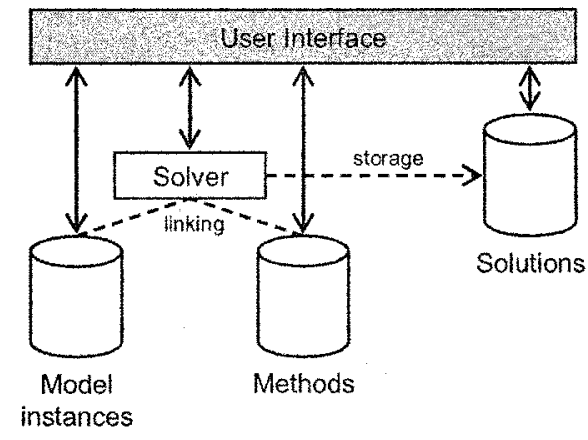

Fig. 1. System architecture.

A method database contains a set of heuristics approaches for solving multiobjective scheduling problems:

1. Priority rules [12], based on the early work of GIFFLER and THOMPSON [11] for generating active schedules.

2. Local search neighborhoods [16] within a multi-point hillclimber.

3. Multi-objective evolutionary algorithms [1], incorporating elitist strategies and a variety of crossover neighborhoods like e.g. uniform order based crossover, order based crossover, two point order crossover, and partially mapped crossover.

4. The 'MOSA' multi-objective simulated annealing algorithm of TEGHEM et al. [19].

5. A module based on the 'AIM' aspiration interactive method [14] for an interactive search in the obtained results.

The model instance database stores the data of the problem instances that have to be solved. General job shop as well as flow shop scheduling problems can be formulated. Besides newly generated data sets, well-known test instances from literature [3] have been included. Solutions are obtained by linking model instances with methods and stored in a solution database. This allows the reuse of specific metaheuristics for a range of problem instances as well as the comparison of results obtained from different heuristic approaches.

A graphical user interface links the modules described above into a single system. Besides the construction of model instances and the definition of metaheuristic search algorithms, an interactive decision making procedure enables the user to select a most preferred schedule. Visualization of alternatives is 
available in alternative space and in outcome space. The alternatives as such are presented as Gantt charts [10], their outcomes as plotted Pareto fronts.

\subsection{Visual interface}

The user interface is a key aspect of the implemented system as it is designed for end users. It brings together visual components to store, retrieve and modify problem instances, configure metaheuristic methods, execute them and manage the obtained results. All components like for example crossover/mutation operators and their corresponding application probabilities are available. New configurations of metaheuristics can be derived from predefined and implemented techniques by simply changing the attribute values of the methods. Also, the progress of the metaheuristics while optimizing a particular problem instance can be monitored by storing the currently best alternatives after definable intervals of evaluations.

After executing methods on problem instances, solutions are obtained that need to be further investigated. As mentioned above, two visualizations are of importance. First, a two-dimensional outcome space plot visualizing the Pareto front. Here, a direct interaction is possible by allowing the user to select alternatives. Second, a visualization of the alternatives as such using job-oriented or machine-oriented Gantt charts [24]. The detailed starting times of the operations can be monitored here, and an indication whether a job is tardy or not is easily available.

In order to allow a widespread use of the software, the graphical user interface is available in English, French, German, Hungarian, Italian, Polish, and Spanish language. Also, a 103-pages printed documentation is available.

\subsection{Optimization and decision making}

The resolution of multi-objective scheduling problems is supported by a twostage a posteriori procedure as described in Section 1. First, Pareto-optimal alternatives or an approximation $P_{a}$ of the Pareto set $P$ are computed using the chosen metaheuristics. Second, an interactive search in the obtained results is performed by the decision maker.

During this interactive decision making procedure, aspiration levels $A=$ $\left\{a_{g_{1}}, \ldots, a_{g_{k}}\right\}$ for each of the optimized objective functions $G(x)=\left(g_{1}(x), \ldots\right.$, $\left.g_{k}(x)\right)$ are chosen. As shown in Figure 2, the elements of the approximation $P_{a}$ of the Pareto set $P$ are accordingly divided into two subsets, the subset $P_{a s}$ of the alternatives fulfilling the aspiration levels $\left(g_{i}(x) \leq a_{g_{i}} \forall i=1, \ldots, k\right)$ and the subset $P_{\neg a s}$ of the alternatives that do not meet the aspiration levels. It is obvious that $P_{a s} \cup P_{\neg a s}=P_{a}$ and $P_{a s} \cap P_{\neg a s}=\emptyset$.

The initial values of the aspiration levels $a_{g_{i}}$ are set to the worst values in $P_{a}: a_{g_{i}}=\max _{x \in P_{a}}\left(g_{i}(x)\right) \forall i=1, \ldots, k$ and as a consequence, $P_{a s}=P_{a}$. The decision maker is allowed to modify the values of the aspiration levels and successively reduce the number of elements in $P_{a s}$ until $\left|P_{a s}\right|=1$. The 


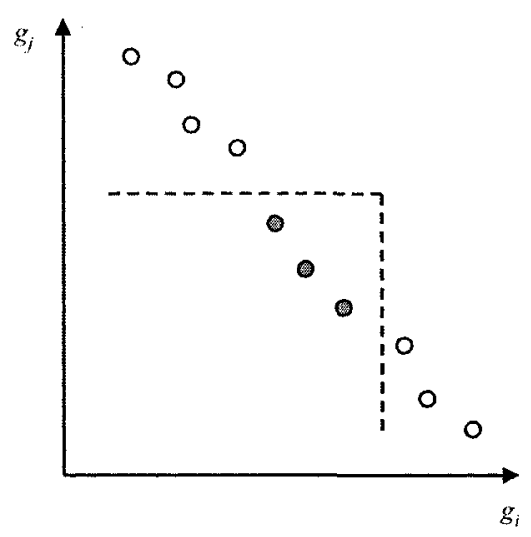

Fig. 2. Decision making component. A cone in outcome space divides the set of alternatives into alternatives fulfilling the aspiration levels (grey background) and alternatives outside the cone (white background).

remaining alternative in $P_{a s}$ is the desired compromising solution $x^{*}$ as the fixed aspiration levels are met by this alternative.

Another interpretation of the method can be seen in a moving cone in outcome space that contains all alternatives fulfilling the current aspiration levels. While the visualization of the outcome space provided by the system is limited to two dimensions at a time, the decision making procedure as such can accommodate higher dimensions without any further problems. The procedure here allows the arbitrary change of the aspiration levels $a_{g_{i}}$ in any direction, enlarging or reducing them. This is important as the situation in which $P_{a s}=\emptyset$ appears, an adjustment of at least one aspiration level $a_{g_{i}}$ is necessary in order to allow the identification of a most preferred alternative $x^{*}$.

\section{Computational results}

Different configurations of the implemented metaheuristics have been tested on benchmark instances of multi-objective machine scheduling problems taken from literature $[1,2]$, ranging from $n=20$ jobs on $m=5$ machines up to $n=100$ jobs on $m=20$ machines. Using various configurations of evolutionary algorithms, close approximations of the true Pareto fronts or the best known solutions could have been obtained.

Algorithm 1 gives the pseudo-code of the applied evolutionary method. It can be seen, that two sets are maintained during search. First, a population $P O P_{i}$ of $n_{p o p}$ individuals, and second, an archive $P_{a}$ of alternatives which are currently not dominated by any other known alternative. After termination of the optimization runs, $P_{a}$ is returned as an approximation of the true Pareto set 
$P$. This strategy allows the evolvement of the population $P O P_{i}$ while keeping the best found alternatives in $P_{a}$, implicitly implementing an elitist strategy.

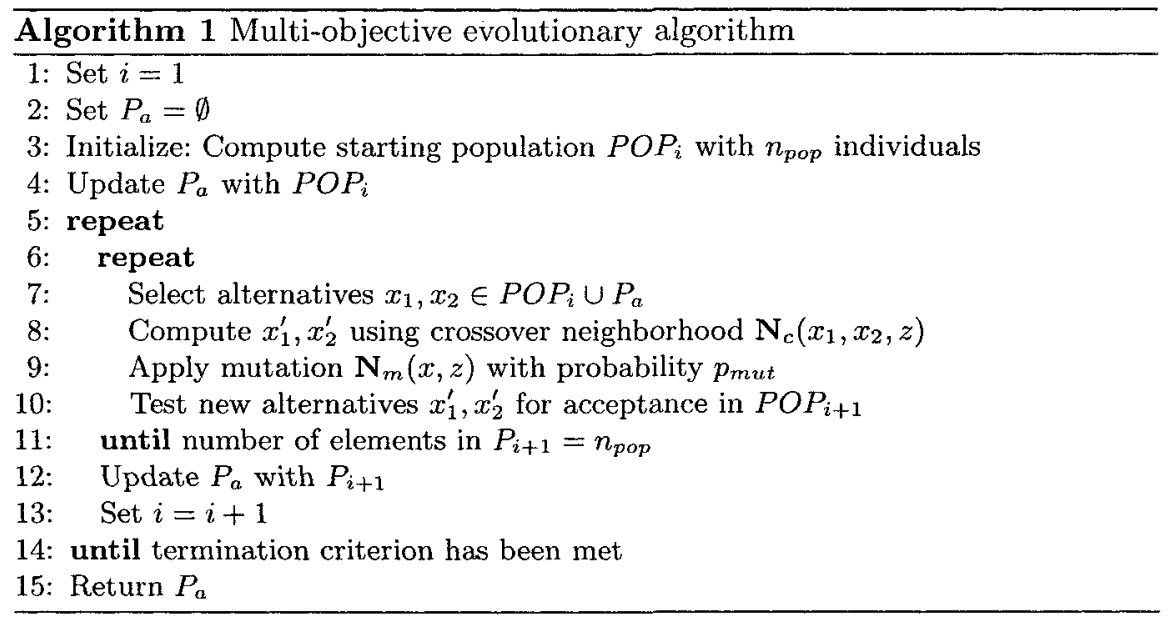

When selecting two alternatives $x_{1}, x_{2}$ for reproduction, the union of both sets $P O P_{i}$ and $P_{a}$ forms a mating pool. Selection is done with respect to the Pareto-ranking-based approach of FONSECA and FLEMING [8]. Crossover operators tested include partially mapped crossover PMX, order based crossover OBX, uniform order based crossover UOBX, and two-point crossover TPOX [22]. New alternatives are generated until a new population $P O P_{i+1}$ has been formed which replaces the old population $P O P_{i}$. Step 10 of Algorithm 1 ensures that no duplicates are added to the succeeding population $P O P_{i+1}$.

The length of the test runs has to be chosen depending on the size of the problem instances. Good termination criteria turned out to be 1,000,000 evaluated alternatives for instances with $n=20,5,000,000$ evaluations for instances with $n=50$, and $10,000,000$ for $n=100$.

For the instances proposed by BASSEUR et al. [2] on the basis of TAILLARD [17], the approximations came close to the best known alternatives of which most have been identified. Unfortunately it was not possible to improve any of them. It may be mentioned however, that for the smaller instances the known results are already proven to be optimal and therefor not further improvable.

New alternatives have been identified dominating the previously reported best known solutions for the instance of BAGCHI [1] with $n=49$ jobs on $m=15$ machines. The considered objective functions of this instance are the minimization of the maximum completion time $C_{\max }$, the minimization of the average completion time of all jobs $\frac{1}{n} C_{\text {sum }}$, and the minimization of the average tardiness of all jobs $\frac{1}{n} T_{\text {sum }}$.

Figure 3 gives a plot of the results in outcome space. The best solutions obtained with a multi-objective evolutionary algorithms using the fitness as- 


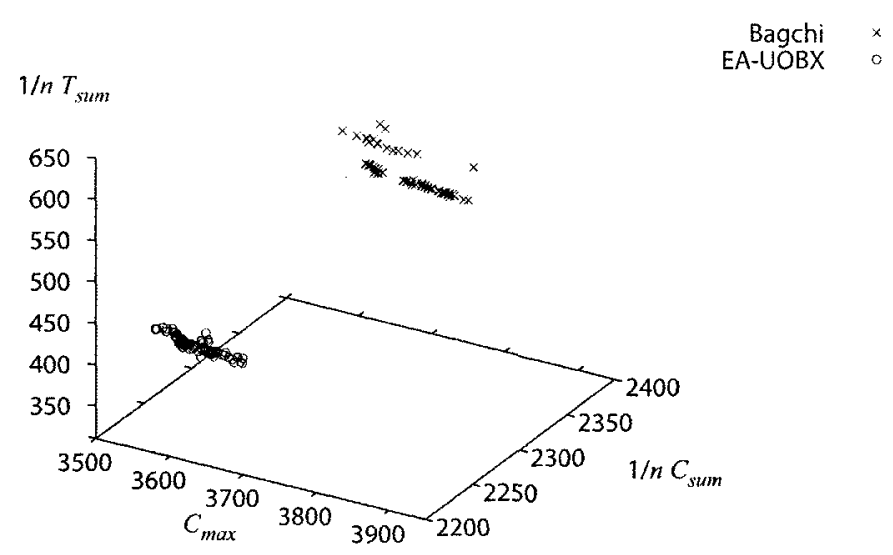

Fig. 3. Comparison of obtained approximation for the problem instance from [1] with previously known best solutions.

signment of FONSECA and FLEMING [8] and a uniform order based crossover UOBX are compared with the results reported by BAGCHI. It can be seen, that all alternatives of [1] are dominated. In particular with respect to the objective of minimizing the average tardiness of jobs, significant improvements have been obtained.

When closer investigating the results, it can be observed that the obtained alternatives are in rather close proximity to each other. The schedules share significant similarities both in outcome space, see Figure 3 , and in alternative space. This indicates that Pareto optimal alternatives are closely concentrated in the search space of feasible alternatives $\mathcal{X}$ and helps to explain to some extend how metaheuristic search may work. As qualitatively good alternatives are typically close to other alternatives of high quality, this information may be exploited when computing neighboring alternatives using crossover or mutation operators.

\section{Conclusions and discussion}

A decision support system for multiple objective scheduling problems has been presented. It incorporates a set of metaheuristics that can be adapted to specific problem instances. As the user interface is highly visual, non-experienced users are able to solve scheduling problems under multiple objectives with comparably little knowledge.

Computational results have been gathered for benchmark instances taken from literature. It has been possible to observe the effectiveness of the implemented methods, even in comparison to the best known results of the test instances. While the results are satisfying with respect to that aspect, a further 
development and improvement of the methods is unfortunately not permitted to the end user as the source code is not accessible.

After an approximation of Pareto-optimal alternatives has been obtained, an interactive decision making module based on the aspiration interactive method allows the identification of a most preferred schedule. The system may also be used to compare different approximation results of various metaheuristic approaches in terms of their approximation quality. It is therefore suitable for demonstrating the use, adaptation and effectiveness of metaheuristics to complex combinatorial optimization problems using the example of machine scheduling under multiple objectives.

The system successfully competed in the European Academic Software Award, a biannual contest of academic software in research and higher education. In this context, it has been evaluated by an international panel of experts. As it is aimed at end users who are not necessarily experts in the relevant field of metaheuristics or scheduling, its' realized concept differs from existing implementations. Rather than being generic like know software class libraries, it is specific. This bears the disadvantage of a potentially difficult adaptation to other problems than scheduling. On the other hand however, as it presents a closed system with no need of adapting and recompiling source code, it may also be used as a demonstration and learning tool in higher education. Based on the experiences gathered, we believe that it is able to stipulate the understanding and use of modern metaheuristics in research and higher education, and contribute to the further development and distribution of modern heuristics.

\section{Acknowledgements}

The author would like to thank Zsíros Ákos (University of Szeged, Hungary), Pedro Caicedo, Luca Di Gaspero (University of Udine, Italy), and SzYMON WILK (Poznan University of Technology, Poland) for providing multilingual versions of the software.

\section{References}

1. Tapan P. Bagchi. Multiobjective scheduling by genetic algorithms. Kluwer Academic Publishers, Boston, Dordrecht, London, 1999.

2. Matthieu Basseur, Franck Seynhaeve, and El-ghazali Talbi. Design of multiobjective evolutionary algorithms: Application to the flow-shop scheduling problem. In Congress on Evolutionary Computation (CEC'2002), volume 2, pages 1151-1156, Piscataway, NJ, May 2002. IEEE Service Center.

3. J. E. Beasley. Obtaining test problems via internet. Journal of Global Optimization, 8:429-433, 1996.

4. J. Błażewicz, K. H. Ecker, E. Pesch, G. Schmidt, and J. Wȩglarz. Scheduling Computer and Manufacturing Processes. Springer Verlag, Berlin, Heidelberg, New York, 2. edition, 2001. 
5. R. W. Conway, W. L. Maxwell, and L. W. Miller. Theory of Scheduling. AddisonWesley, Reading, MA, 1967.

6. Richard L. Daniels. Incorporating preference information into multi-objective scheduling. European Journal of Operational Research, 77:272-286, 1994.

7. Richard L. Daniels and Joseph B. Mazzola. A tabu-search heuristic for the flexibleresource flow shop scheduling problem. Annals of Operations Research, 41:207230, 1993.

8. Carlos M. Fonseca and Peter J. Fleming. Genetic algorithms for multiobjective optimization: Formulation, discussion and generalization. In Stephanie Forrest, editor, Proceedings of the Fifth International Conference on Genetic Algorithms, pages 416-423, San Mateo, CA, 1993. Morgan Kaufmann Publishers.

9. Tomáš Gál, Theodor J. Stewart, and Thomas Hanne, editors. Multicriteria Decision Making: Advances in MCDM Models, Algorithms, Theory, and Applications, volume 21 of International Series in Operations Research 8 Management Science. Kluwer Academic Publishers, Boston, Dordrecht, London, 1999.

10. Henry L. Gantt. Efficiency and democracy. Transactions of the American Society of Mechanical Engineers, 40:799-808, 1919.

11. B. Giffler and G. L. Thompson. Algorithms for solving production-scheduling problems. Operations Research, 8:487-503, 1960.

12. R. Haupt. A survey of priority rule-based scheduling. Operations Research Spektrum, 11(1):3-16, 1989.

13. Sang M. Lee and David L. Olson. Goal programming. In Gál et al. [9], chapter 8, pages 8.1-8.33.

14. V. Lotfi, T. J. Stewart, and S. Zionts. An aspiration-level interactive model for multiple criteria decision making. Computers $\&$ Operations Research, 19(7):671681, 1992.

15. Michael Pinedo. Planning and Scheduling in Manufacturing and Services. Springer Verlag, Berlin, Heidelberg, New York, 2005.

16. Colin R. Reeves. Landscapes, operators and heuristic search. Annals of Operations Research, 86:473-490, 1999.

17. Eric Taillard. Benchmarks for basic scheduling problems. European Journal of Operational Research, 64:278-285, 1993.

18. Vincent T'kindt and Jean-Charles Billaut. Multicriteria Scheduling: Theory, Models and Algorithms. Springer Verlag, Berlin, Heidelberg, New York, 2002.

19. E. L. Ulungu, J. Teghem, P. H. Fortemps, and D. Tuyttens. MOSA method: A tool for solving multiobjective combinatorial optimization problems. Journal of Multi-Criteria Decision Making, 8:221-236, 1999.

20. David A. Van Veldhuizen and Gary B. Lamont. Multiobjective evolutionary algorithms: Analyzing the state-of-the-art. Evolutionary Computation, 8(2):125-147, 2000 .

21. Philippe Vincke. Multicriteria Decision-Aid. John Wiley \& Sons, Chichester, New York, Brisbane, Toronto, Singapore, 1992.

22. Darrell Whitley. Permutations. In Thomas Bäck, David B. Fogel, and Zbigniew Michalewicz, editors, Handbook of Evolutionary Computation, chapter C3.3.3, pages C3.3:14-C3.3.20. Institute of Physics Publishing, Bristol, 1997.

23. Andrzej P. Wierzbicki. Reference point approaches. In Gál et al. [9], chapter 9, pages $9.1-9.39$.

24. James M. Wilson. Gantt charts: A centenary appreciation. European Journal of Operational Research, 149:430-437, 2003. 